\title{
Coding Theory Adapted to Gait Perception
}

\author{
James E. Cutting \\ Cornell University
}

\begin{abstract}
Restle's coding theory for movement perception is merged with certain aspects of Johansson's vector descriptions of movement to investigate the relative perceptual prominence of several kinds of displays of human walkers. Two versions of a coding model are entertained. The first considers the results of a demonstration and an experiment in which points of light are mounted in either of two ways: on major joints of the body or off them. Results suggest that on-joint displays are the better figures but that off-joint displays are fully adequate for representing human motion. Vector codes and a system for relating them are developed to reflect these results. This first model, however, is inadequate for a second demonstration in which the spatial relations among the lights are scrambled. The model's problem lies in the fact that it does not discriminate between a canonical walker display and the spatially anomalous one. Thus, the model is elaborated into a second form to account for the difference. It incorporates what my colleagues and I have called centers of moment. The force of this exercise is to demonstrate the necessity for movements and spatial extent to be coded interactively. Only in this way can a coding theory work in the domain of gait perception.
\end{abstract}

Johansson (1973) demonstrated that the actions of human beings could be identified from the movements of lights mounted on the major joints of the body. That is, with the surround darkened, the flow pattern of these lights is sufficient to determine the presence of an individual painting a wall, bicycling, walking, running, or doing pushups. This technique is not new-it is, in fact, over a century old (see Marey, 1895/1972)but Johansson was the first to apply it to a study of complex event perception.

Following Johansson's lead, my colleagues and $I$ have begun to explore the perception of these and other complex displays. The beauty of schematic, complex displays lies in the fact that one can formalize the mathematics of their motion and perhaps their perceptions as well. With this goal in mind,

This research was supported by National Institutes of Health Grant MH 35530 to the author and was made possible by the psychology department of Stanford University, where the author spent a sabbatical/leave from Wesleyan University.

I thank Carol Krumhansl, Dennis Proffitt, and Frank Restle for their comments and Carl Latkin for his aid with Experiment 1.

Requests for reprints should be sent to J. Cutting, Department of Psychology, Uris Hall, Cornell University, Ithaca, New York 14853. we have explored not only the perception of gait (Cutting, 1978a; Cutting \& Kozlowski, 1977; Cutting, Proffitt, \& Kozlowski, 1978) but also the perception of rolling motion (Proffitt \& Cutting, 1979; Proffitt, Cutting, \& Stier, 1979) and the perception of aging faces (Cutting, 1978b). In each of these domains, and in several others (Cutting \& Proffitt, in press), we have suggested that perception is guided by an abstract entity. We call it the center of moment, and it is a point in the geometric space of the object around which all points within the structure have systematic reference. Its locale for a walker determines gender, its locale for a cluster of lights mounted on a wheel determines how wheellike that structure will appear when rolling, and its locale for a schematic profile of a human face determines how well certain transformations of that profile will generate younger- and older-looking people.

One problem with our previous accounts of event perception and centers of moment is that they are not very rigorous. In general, we have offered no formal specification of what we mean by a "point around which everything moves," and in particular we have not formally specified nested relations 
in the dynamic array. The brunt of this article is to render these notions more formal. In particular, I will adapt a coding theory of motion perception to the perception of gait and gait-related displays.

Recently, Restle (1979) presented a coding theory for the perception of moving clusters of lights. It is an adaptation of Leeuwenberg's $(1971,1978)$ theory for the perception of static forms and line drawings. Restle proposed that the perceived coherence in a dynamic display can be captured formally by the measurement of the number of shared parameters among the moving lights. The power of his formulation is that possible perceptual interpretations can be ordered in terms of prominence, that is, the likelihood that the perceptual system imposes such particular organization. This is done by comparing the information loads of the various perceived forms-roughly, counting the number and nestings of various parameters of movement-with the potential information load of the display considered as if it had no shared parameters. In short, the more that it shared, the more prominent the perceived form.

Restle's (1979) coding model is most elegant for considering the simple motion of a few points. It seems to lose its elegance, though not its power, when the motions of many lights are considered. But it loses its generalizability when those lights do not move in a circular pattern. Of course, Restle made no attempt to apply his coding model to demonstrations other than those of Johansson (1950) and a few related phenomena, and thus he cannot be faulted for not applying his model to Johansson's later demonstrations. Nonetheless, in a domain like gait, in which the motions are primarily pendular and not degenerations of circular patterns, application of his coding model cannot be straightforward. To retain a certain degree of simplicity while adapting it to the complex patterns of human locomotion, changes must be made. It should come as no surprise that those suggested here ultimately include the center of moment. They are also entirely consistent with the ideas of Johansson (1958, 1977; Johansson, von Hofsten, \& Jansson, 1980).

A five-step approach will be taken toward an understanding of motion perception in gait and gait-related displays. Since codingtheory analysis begins with what is seen, the first step is a demonstration assessing a particular gait pattern and configuration not used in previous studies. The second step is an experiment comparing this novel configuration with configurations more similar to those used previously. The third step involves the presentation of a version of a coding model to account for the data in the first demonstration and the experiment. The fourth step entails a second demonstration, with another novel gaitlike pattern and configuration. This forces a fifth step, where I present a revised coding model and apply it to all data on gait.

\section{Demonstration 1: Identification of a} Walker With Lights Mounted off Joints

There is some dispute concerning one aspect of gait perception. A number of colleagues have told me that when points of lights are mounted off the major joints of the body, the presence of a human being is not seen in the dynamic display. This struck me as curious, if not implausible. The roots of the claim are not clear, but Johnson-Laird and Wason (1977), for example, wrote that to perceive a human walker, one theory

predicts that lights at major joints would facilitate this process, whereas lights in the middle of the limbs would not, and indeed Johansson reports that the latter do not give rise to the perception of a man walking. (p. 343)

They refer to Johansson (Note 1), but I can find no mention of such a result there or in Johansson (1973). Regardless, it seemed to me that a demonstration using such a display would be a good place to begin a coding theorylike analysis because it provides an interesting, alternative structure to the gait stimuli used previously.

\section{Method}

The dynamic stimulus was generated by a FORTRAN program displayed on a Tektronix 604 monitor display scope and driven by a Data General Nova computer. (Instrumentation and listing of that program are given in Cutting, $1978 \mathrm{c}$, and a more complete description of the parameters involved may be found in Cutting, 1978a.) In essence, the program generates moving clusters of lights that mimic the movements of a human 
walker; it is as if the points of light were mounted on the major joints of an individual as she or he walks from left to right across a dark stage. In the original program, lights represented movements of the right shoulder and the right hip, and the left and right elbows, wrists, knees, and ankles. Occlusions of lights occurred at natural places within the step cycle. An 11 th light was also present, as if mounted on the head. The head, of course, is not a joint, and Johansson (1973), Cutting and Kozlowski (1977), and Kozlowski and Cutting (1977) demonstrated that the head is not necessary to these displays. Nevertheless, it was added to make the stimuli look more natural.

The core program was modified to display lights as if mounted halfway between the major joints. This reduced the total number of lights to seven: one on the right biceps, one on each of the forearms, one on the right thigh, one on each of the calves, and one on the head. In addition, a ground line was added, on which the stimulus "walked." This stimulus with ground line is shown in schematic form in the lower panel of Figure 1. Of course, the figure outline was not present in the display.

This one stimulus was videotape-recorded and shown to 10 viewers en masse as part of a course project. Visual angle was between $2^{\circ}$ and $4^{\circ}$. These viewers were from the Wesleyan University Graduate Summer School, had no experience at viewing point-light displays, and were given no instructions except simply to write down what they saw in the display. The stimulus was on screen for $3.6 \mathrm{sec}$, taking six steps.

\section{Results and Discussion}

All 10 viewers reported seeing a person walking. Later, when I replayed the stimulus and asked the viewers where the lights were mounted, all reported that the lights were mounted on the body. When asked to be more specific, the viewers responded in mixed manner. Some suggested that lights were mounted on joints, some gave responses suggesting that lights were mounted on fleshy areas of the body, and others responded that some lights were mounted on and some off the joints. None suggested that the display was anything other than a real human being, and all expressed surprise that it was computer generated.

Probed further about the movement of the arms and legs, most viewers reported a normal bending of arms and legs. This final aspect of the percept is theoretically important. From statements by Johansson (1973, 1975), one would have to assume that he would claim that lights mounted only on the biceps and forearm would be seen as a piston oscillating in phase as a pendulum. Given lights mounted elsewhere on a walker, how-
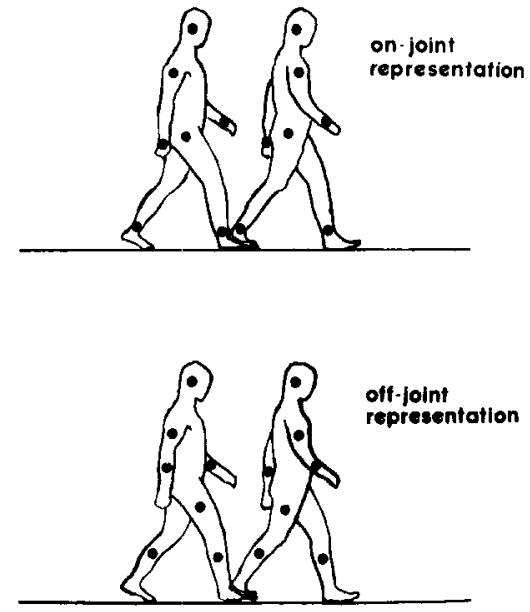

Figure I. Schematic representations of a male walker. (In the upper panel, lights are mounted on the head, on the right shoulder and hip, and on the right and left wrists and ankles. In the dynamic displays only the lights are seen. This is called an on-joint representation of a walker. In the lower panel, lights are mounted on the head, on the upper right arm and leg, and on the lower right and left arms and legs. These lights are halfway between major joints, and the configuration is called an off-joint representation of a walker. Notice that the two types of stimuli have the same number of lights.)

ever, the robustness of the perception of a human form appears to supervene this type of perception. It is seen clearly as a swinging arm, not a swinging piston. This is undoubtedly due, in part, to overlearning and general familiarity with human movement.

The extreme form of the claim put forth by Johnson-Laird and Wason (1977) is incorrect: A human form can be seen in a dynamic display where points of light have been mounted off the joints. However, it remains possible, in fact likely, that some less extreme form of the claim is true. Perhaps displays with lights mounted off joints are less convincing as stimuli than are those with lights mounted on joints.

If I were to ask viewers simply to compare on- and off-joint displays I was afraid that demand characteristics and other extraneous variables might intercede. Thus, rather than taking such a direct approach, I chose an indirect one. My previous use of these stimuli had been primarily in asking viewers to make gender judgments of the displays, that is, indicating which walkers were male and which were female (Cutting, 1978a). Thus, 
I decided to ask viewers to make such judgments of on-joint and off-joint displays that were programmed to emulate male and female gaits. From these results I would make inferences concerning the general goodness of form in these displays.

\section{Experiment 1: A Comparison of On-Joint and Off-Joint Displays}

\section{Method}

The base algorithm of Cutting (1978c) was used. The most malelike and the most femalelike movements were chosen from those used by Cutting (1978a). These displays differ in the locus that generates the movements. Practically speaking, this means that they differ in the amount of shoulder swing as compared to hip swing. Ratios of these two movements for the male walker was $4 / 1$ and for the female 1/4. Changes in the movements of shoulder and hip cause changes in the movements of all other points in the display.

Off-joint displays were generated as discussed in the previous demonstration. On-joint displays were similar in that they had the same number of lights, but the lights were mounted on the joints of the body. That is, they were placed as if they were on the right shoulder, left and right wrists, right hip, left and right ankles, and the head. No lights were on the elbows or knees, and as suggested before, these are unnecessary to perceive the motion of arms and legs. Both types of stimuli are shown in schematic form for a male walker in Figure 1. Ground lines were present for both stimulus types.

Two videotape sequences were prepared. Each consisted of 16 stimuli: 2 types of stimuli (on and off joints) $\times 2$ genders (male and female) $\times 4$ repetitions of each item, with $8 \mathrm{sec}$ between trials. Sequence $1 \mathrm{con}$ sisted of a randomization of all 16 items, and Sequence 2 consisted of two blocks of eight stimuli, in which all eight of one stimulus type were segregated from the other.

Three groups of viewers participated in the study. Group 1 consisted of 116 students of introductory psychology at Wesleyan University participating as part of a course project. These viewers were selected from a class of 270 and consisted of all students in the first seven rows of the lecture hall who could see the video monitor from a viewing angle of not less than $60^{\circ}\left(90^{\circ}\right.$ being directly in front of the monitor). Visual angle for stimuli varied from about $1 / 2^{\circ}$ to $2^{\circ}$. This range is comparable to that of Kozlowski and Cutting (1977, Experiment 5). En masse, these viewers were presented first a familiarization sequence showing lights mounted on all joints. These 11-light configurations will be called canonical stimuli. Viewers were then shown Sequence 1.

Group 2 consisted of 10 Wesleyan University undergraduates who were paid to participate in a more controlled setting. Singly or in pairs, they sat directly in front of a video monitor, with a stimulus visual angle of $3^{\circ}-4^{\circ}$. Like Group 1, all were presented first with a familiarization sequence of canonical stimuli. Then they were presented Sequence 2 so that half viewed the on- joint stimuli before the off-joint stimuli, and half viewed them in reverse order.

Group 3 consisted of the 10 viewers from Demonstration 1. After viewing the demonstration item several times, they viewed, en masse, the off-joint block of Sequence 2 before the on-joint block.

\section{Results and Discussion}

The judgments of gender were generally more accurate for on-joint stimuli than for off-joint stimuli. This pattern is apparent for all three groups, as shown in Table 1.

These results were perhaps most clear for Group 1. Gender of on-joint stimuli was accurately determined on $65 \%$ of all trials, $t(115)=8.6, p<.001$, whereas gender for off-joint items as determined on only $56 \%$ of all trials, $t(115)=3.77, p<.001$. This difference was reliable, $t(115)=3.9, p<$ .001 . However, the mixture of the two types of stimuli within one test order produced an unanticipated result. Many viewers, perhaps unable to make gender judgments of the kind that I asked, seemed arbitrarily to assign the on-joint stimuli to one gender category and the off-joint stimuli to the other. Since the overt demand of the experiments was to judge gender, and since the on-joint/offjoint distinction was certainly more salient than the male/female distinction, many viewers appeared to comply by parsing the stimulus domain according to the most visible distinction. Luckily, assignments of offjoint stimuli to males and on-joint stimuli to females were about as frequent as the reverse configuration, but results still suggest regression toward the mean.

Group 2, then, saw the on-joint and offjoint sequences in counterbalanced order. Their results show better performance and the same general superiority of the on-joint stimuli: The gender of on-joint stimuli was correctly determined on $71 \%$ of all trials, $t(9)=2.68, p<.02$, and that for off-joint stimuli was determined on only $65 \%, t$ $(9)=2.07, p<.07$. Unlike that for Group 1 , however, this difference was not statistically reliable.

The question remains, however, as to whether off-joint stimuli would be accurately judged if the viewers had not had prior experience with point-light stimuli. In other words, all viewers in the first two groups had 
Table 1

Percentage Correct Identification of Computer-Generated Male and Female Walkers

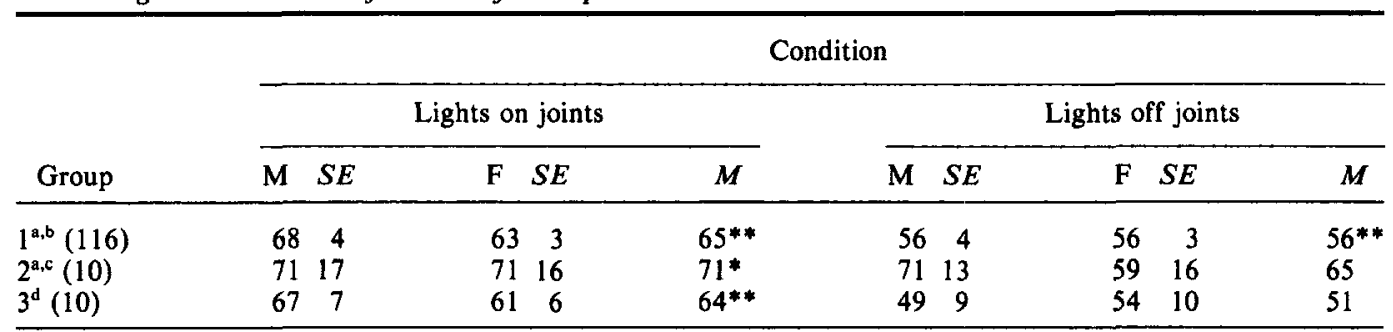

Note. $\mathrm{M}=$ male, $\mathrm{F}=$ female, $S E=$ standard error. Numbers in parentheses are $n \mathrm{~s}$.

"Test preceded by exposure to canonical stimuli (those with lights on all major joints).

${ }^{b}$ On-joint and off-joint stimuli presented randomly intermixed within same test sequence.

${ }^{c}$ On-joint and off-joint stimuli separated into blocks, with block order counterbalanced.

"Viewers had no prior experience with these stimuli. The off-joint condition preceded the on-joint condition.

$*=p<.05,{ }^{* *}=p<.01$.

seen canonical stimuli, with lights mounted on all joints, before judging on- and off-joint stimuli. It could well be that the off-joint representations would not be sufficient for the representation of gender if viewers had not seen the canonical stimuli before them. Group 3, then, viewed the off-joint stimuli before the on-joint stimuli. These viewers correctly determined the gender of off-joint displays on only $51 \%$ of all trials $(t<1)$, whereas they judged correctly the on-joint configurations on $64 \%$ of all trials, $t(9)=$ $3.3, p<.01$.

The overall pattern of results is clear. Removing the elbow and knee lights from the canonical displays interfered little with viewers' ability to make gender judgments. This result is consistent with those of Kozlowski and Cutting (1977, Experiment 5), who found that, in general, no pair of lights was necessary to the display and that nearly any pair was sufficient. Moving all lights off joints, however, did interfere with gender identifiability. In fact, when viewers have no experience at perceiving these displays, they appear not to be able to make gender judgments at all.

Notice the "male" response bias in onjoint conditions for Groups 1 and 3 and in the off-joint condition for Group 2. These patterns are consistent with those found previously. Cutting (1978a) reported that this computer algorithm seemed somewhat more successful at generating male gait than female gait. Since this bias seems to occur for all types of stimuli and is orthogonal to my concerns, it does not detract from the main effect of gender identification as discussed in this section.

In summary, then, these results support an inference about the perception of the stimuli: Insofar as accuracy and consistency of gender judgments can be taken as an index of goodness of figure in these dynamic displays, on-joint displays are better stimuli than are the off-joint displays. Why this may be so is pursued in the following section, in which I introduce a variant of Restle's (1979) coding model.

\section{A First Model: Vector Codes}

\section{Johansson and Restle for a Rolling Wheel}

The crux of Johansson's analyses of movement is that common motion vectors are extracted from a dynamic display containing components of a figure that move in complex ways. Consider a case from the perception of rotary motion in which two lights are mounted on a wheel, one at the center and one on the perimeter. The path of the light at the center will describe a straight line as the wheel rolls across a flat surface. The light on the perimeter will describe a cycloid and can be most easily considered as composed of two vectors, the translatory vector and the rotational vector of the wheel. According to Johansson $(1973,1975)$, since the two lights share the translatory (or straight-line) vector 
of the wheel, and since the residual of the cycloidal motion of the perimeter light is a rotational vector, a rolling wheel is seen.

Johansson states that a rolling wheel is seen because the visual system extracts the common vector first - that of translationand then perceives residual motion as indicating the object's action and form - a rolling wheel. Wallach (1965) has suggested the same two-step process but in reversed order. $\mathrm{He}$ contended that object-relative motion is seen first (one light moving circularly about the other) and angular displacement second (the group of lights moving across the visual field). Elsewhere evidence has been presented in support of Wallach's view (Proffitt \& Cutting, 1979; Proffitt et al., 1979). For this discussion, however, the results do not detract from Johansson's insight: The movement of lights mounted on an object can be thought of as generated through vector addition; perception of the object represented only by these lights is accomplished through vector decomposition. One aspect of Johansson's approach that appears problematic is that he does not tell us how the perceptual system decomposes complex vectors in the appropriate way, except through an appeal to simplicity, projective geometry, and the fundamental three-dimensionality of space. In particular, it is not a trivial problem as to how one should arrive at a common vector first, without appeal to residual components, nor is it always clear what constitutes simplicity (Sober, 1975).

Restle's (1979) coding theory of pure movement shares certain features with Johansson's approach. Its strength is that it operationalizes Johansson's (1950, 1973) concept of simplicity. It claims that moving clusters of lights are seen as coherent entities rather than as a conflation of random lights because their coherence is based on shared parameters of movement, all based on circular motions. When coding movement into component parameters, the description of a rolling wheel, for example, would need fewer free variables than would the description of two lights moving independently, one in cycloidal and the other in linear fashion. Restle's argument is that the perceptual system chooses a description of the stimulus that is most parsimonious when compared to the total possible variables that might have been used for specifying the movements of all lights.

Two problems. Restle's model is restricted to motions generated on a circle. As suggested earlier, pendular motions, such as those seen in human body movements, pose somewhat of a problem. For example, his five parameters-phase, amplitude, rate, axis of movement, and tilt-could apply equally well to pendular motion. Thus, although one could use Restle's system for pendula or for circles, one could not discriminate between them without providing some higher order code for the type of movement domain, pendular or circular. Moreover, these are not the only two domains to which these five parameters could apply, they are just mechanically the most obvious. One could just as easily apply them to movements of a light along a square-shaped path (see Johansson, 1975), a triangular one, or any other. Only our imagination limits us in this domain.

A second problem for Restle's current formulation, though not as serious, is translation. His model provides no simple way to code nonrepeating movement. Coding theory is certainly flexible enough to add such parameters for rate and direction of translation, but within Restle's (1979) model it is not clear where and how one would do this. This is important because the number of variables necessary to code this movement would have direct influence on the absolute (though not relative) prominence value of the perceived configuration.

\section{A Hybrid Approach for Human Walkers}

Despite coding problems of movement domain and of translation, I have found Restle's (1979) approach useful in formulating a view about the gait research begun by Johansson. From Johansson I will take the approach of using rather complex vectors as primitives in my description. That is, I will not attempt to decompose movements further than the general motions performed by the various parts of the human body. From Restle I will take the approach of his coding theory that assumes that the perceptual system extracts the most parsimonious descrip- 
tion of movements. This hybrid approach allows the computation of relative prominence values of perceived configurations without determining the hundreds of parameters that make up human movement.

Consider one view of walking. By extension from the example of a rolling wheel, the walker has a vector of translation with an additional component of undulation, an up and down movement attributed to the varying height of the hips during the step cycle. This periodic, wavy pattern is the common vector that underlies all movement in the head, arms, legs, shoulders, and hips. Thus, points of light mounted on the joints of the body share a common fate whose vector path is the undulation and translation on walking across a flat surface. Pendular motions of arms and legs are superimposed on this common vector and make more complex paths.

One can assume that when considering onand off-joint representations of walkers, Johansson would point to the difference in residual movements of the lights in the two conditions. In the on-joint figures, the movements of the residuals would be pure pendula, whereas in the off-joint figures, those movements would be combinations of vectors of different pendula. To capture this notion I propose a vectorlike description for synthetic on-joint and off-joint representations of walkers. The notational system used takes the following general, simple form: $\mathrm{L}=\mathbf{M}(x)$, where L stands for the particular light under consideration and $\mathbf{M}(x)$ represents the motion of a particular point. $\mathbf{M}(x)$ can be any complex vector and is used as a primitive within this system. Thus, the equation reads: The movement of $\mathrm{L}$ is described wholly by the movement of $x$. Additions of movement will appear in the right-hand side of many equations. These signify vector additions.

For simplicity's sake, consider the lights representing only the right side of the body of a synthetic walker who is walking from left to right at uniform velocity across a flat surface. These lights are shown in the upper left panel of Figure 2 for the on-joint stimulus and numbered $1-5$ as representing the head, shoulder, wrist, hip, and ankle, respectively. For the off-joint stimulus, they are shown in the upper-right panel and num-

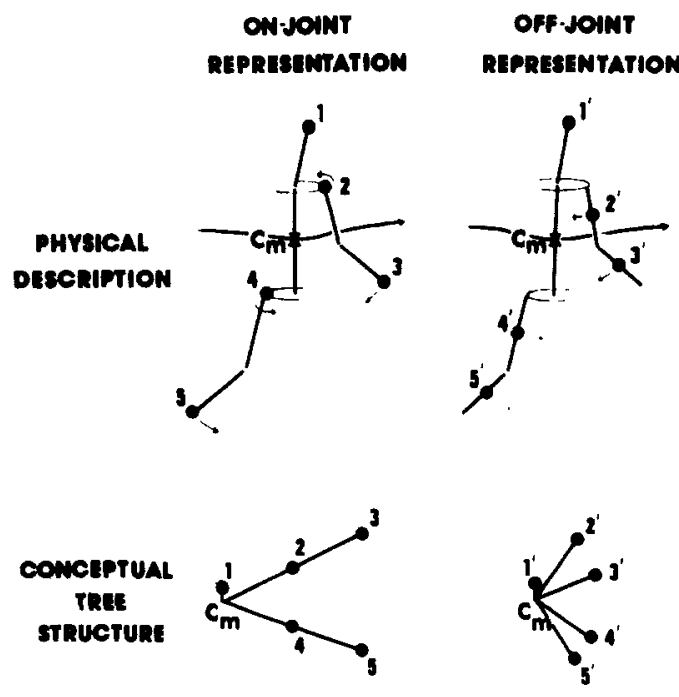

Figure 2. In the upper panels are the physical, vectorlike descriptions of the on- and off-joint walkers, considering only the right side of the body; below these are conceptual tree structures representing the nesting of movements. (Notice that both figures have centers of moment.)

bered $1^{\prime}-5^{\prime}$ for the head, upper arm, lower arm, upper leg, and lower leg, respectively. Vector additions and substitutions requisite for this type of analysis are shown in Table 2. Scrutiny of both Figure 2 and Table 2 will be necessary for the account that follows.

\section{Coding of an On-Joint Stimulus}

Consider first the lights and their vector paths for the on-joint stimulus. The movement of the head, at least in the synthetic versions used in the preceding experiment and demonstration, can be described by simply noting that it follows the same vector path of the highest order center of moment, $C_{m}$. This center of moment lies between the shoulders and hips, and its relative location is proportional to the widths of the walker's shoulders and hips (Cutting et al., 1978).

Two points are worth noting here. First, the vector path of the center of moment is complex and composed of at least three simple vectors - uniform translation, a horizontal oscillation that contributes a lunging motion, and near-vertical oscillation (or undulation). However, since this account allows use of rather coarse-grained movements 
Table 2

Vector Analyses for Computer-Generated Walkers

On-joint walker: $I_{i}=13$

Off-joint walker: $I_{i}=15$

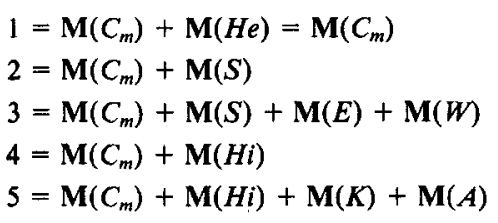

$\mathbf{s}=\mathbf{M}\left(C_{m}\right)+\mathbf{M}(H i)+\mathbf{M}(K)+\mathbf{M}(A)$

$1^{\prime}=\mathbf{M}\left(C_{m}\right)+\mathbf{M}(H e)=\mathbf{M}\left(C_{m}\right)$

$2^{\prime}=\mathbf{M}\left(C_{m}\right)+\mathbf{M}(S)+\mathbf{M}(E) / 2$

$\mathbf{3}^{\prime}=\mathbf{M}\left(C_{m}\right)+\mathbf{M}(S)+\mathbf{M}(E)+\mathbf{M}(W) / 2$

$4^{\prime}=\mathbf{M}\left(C_{m}\right)+\mathbf{M}(H i)+\mathbf{M}(K) / 2$

$\mathbf{S}^{\prime}=\mathbf{M}\left(C_{m}\right)+\mathbf{M}(H i)+\mathbf{M}(K)+\mathbf{M}(A) / 2$

Reduced code by vector

substitution: $I_{s}=7$,

Reduced code by vector

substitution: $I_{s}=11$,

$$
\begin{aligned}
\mathbf{P} & =f\left(I_{i} / I_{s}\right) \\
& =f(1.86)
\end{aligned}
$$

$$
\begin{aligned}
\mathrm{P} & =f\left(I_{/} / I_{s}\right) \\
& =f(1.36)
\end{aligned}
$$

$1=\mathbf{M}\left(C_{m}\right)$

$2=1+\mathbf{M}(S)$

$3=2+\mathbf{M}(E)+\mathbf{M}(W)$

$4=1+\mathbf{M}(H i)$

$5=4+\mathbf{M}(K)+\mathbf{M}(A)$

$$
\begin{aligned}
& 1^{\prime}=\mathbf{M}\left(C_{m}\right) \\
& 2^{\prime}=1^{\prime}+\mathbf{M}(S)+\mathbf{M}(E) / 2 \\
& 3^{\prime}=1^{\prime}+\mathbf{M}(S)+\mathbf{M}(E)+\mathbf{M}(W) / 2 \\
& 4^{\prime}=1^{\prime}+\mathbf{M}(H i)+\mathbf{M}(K) / 2 \\
& 5^{\prime}=1^{\prime}+\mathbf{M}(H i)+\mathbf{M}(K)+\mathbf{M}(A) / 2
\end{aligned}
$$

Note. $\mathbf{M}=$ movement of the point that follows it; $C_{m}=$ highest order center of moment; $\mathrm{He}=$ head, $S=$ shoulder; $E=$ elbow; $W=$ wrist; $H i=$ hip; $K=$ knee; $A=$ ankle. Numbers refer to the movements of lights shown in Figure 2. $I_{i}=$ total number of vectors needed to specify the lights independently; $I_{s}=$ total number of vectors needed to specify the lights as a coherent system. $\mathrm{P}$ is the prominence of the perceived configuration as a function $(f)$ of $I_{i}$ divided by $I_{s}$. See also Footnote 1 .

as primitives within the system, no further decomposition is necessary. Moreover, in this account it is the sharing of vectors that is foremost, and since, in my view and that of my colleagues, all of the body shares the movement of this one point, it will serve to consider it simply as a single vector. Second, the head usually sways from side to side during gait (see Murray, 1967) and therefore when projected onto the sagittal plane, should bob up and down slightly within the step cycle over and above the general undulation of the body. This bobbing movement, however, is slight when seen from the side, was omitted from the computation of movement, and thus will be omitted from this analysis. Thus, for the light mounted on the head, $1=\mathbf{M}\left(C_{m}\right)+\mathbf{M}(\mathrm{He})$, or since the additional computed movement of the head is null, $1=\mathbf{M}\left(C_{m}\right)$. In this manner, the movement of the light on the head, 1 is entirely described by the movement of the $C_{m}$, or highest order center of moment.

The movement of the light on the right shoulder, 2 , is composed of two complex vectors. First, it shares with the head (and all other points) the movement of the $C_{m}$. Added to this is the vector of the shoulder. This movement is described by Cutting (1978a) as a counterclockwise elliptical movement due to the oscillation of the torso, which twists against itself in the manner of a flat spring. Thus, $2=\mathbf{M}\left(C_{m}\right)+\mathbf{M}(S)$. That is, the movement of the light at the right shoulder is composed of the movements of two vectors, that of the highest order center of moment and that of the shoulder.

The movement of the light on the right wrist, 3 , is composed of four vectors: that of the center of moment and the shoulder, plus the additional movements of the (unseen) elbow and the wrist. Both the elbow and the wrist movements are pendular, moving respectively around the shoulder and the elbow. Thus, $3=\mathbf{M}\left(C_{m}\right)+\mathbf{M}(E)+\mathbf{M}(W)$, where $E$ and $W$ stand for the elbow and the wrist.

The movements of the lights mounted on the hip and ankle are determined in a similar manner to that of the shoulder and the wrist, where $H i, K$, and $A$ stand for hip, knee, and ankle, respectively. 
The movements of these five lights in terms of their vector composition are shown in the upper left panel of Table 2. For purposes of comparison consider next the vectors for an off-joint set of lights, as shown in the upper-right panel of Table 2.

\section{Coding of an Off-Joint Stimulus}

The light on the head is, of course, the same in the off-joint version as it is in the on-joint version. Thus, its vector description is the same. However, this is the last identity between the two types of stimuli.

The movement of the light on the upper arm, mounted exactly halfway between the shoulder and the elbow, can be described as composed of three complex vectors: the movement of the highest order center of moment, the movement of the shoulder, and half of the movement of the elbow. That is, since the light is mounted halfway down the upper arm pendulum, its excursion is half that of the elbow, which lies at the end of that pendulum. Thus, $2^{\prime}=\mathbf{M}\left(C_{m}\right)+\mathbf{M}(S)$ $+\mathbf{M}(E) / 2 .^{1}$

The movement of the light on the lower arm is composed of four complex vectors: those of the highest order center of moment, shoulder, elbow, and half of the wrist. Thus, $3^{\prime}=\mathbf{M}\left(C_{m}\right)+\mathbf{M}(S)+\mathbf{M}(E)+\mathbf{M}(W) / 2$. In an analogous manner, the composite vectors are determined for the movements of the lights mounted on the upper leg $\left(4^{\prime}\right)$ and lower leg $\left(5^{\prime}\right)$. These are shown in Table 2.

\section{Reducing Codes to Minimal Form by Vector Substitution}

The differences between the on-joint and off-joint versions of walkers become more apparent when shared vectors are substituted into the descriptions in the upper panels of Table 2. Notice that for the onjoint representations, $2=1+\mathbf{M}(S)$. That is, the movement of Light 2 (mounted on the shoulder) is the same as that of Light 1 , with the addition of the $S$ vector, the movement of the shoulder. In turn, the movement of Light 3 can be described simply as the movement of Light 2 plus the vectors $E$ and $W$, the movements of the elbow and wrist. In a similar manner, the leg vectors can be combined to simplify the description.
Not so with the off-joint representation. As shown in Table 2, little vector substitution can proceed and then only with the substitution of the vector underlying Light 1 at the head. This light has the same movement as the highest order center of moment.

Following the general form of Restle's (1979) coding theory, one can determine the information load of the movement of the walkers. In my adaptation of the theory, this $\left(I_{l}\right)$ is the number of primitive vectors needed to specify the movements of all lights if they are considered as independent. For an onjoint walker, $I_{i}=13$, since 13 vectors are specified in the upper left panel of Table 2. The prominence $(P)$ of any given perceptual interpretation of any given configuration of moving lights is a function of $I_{i}$ divided by the total number of parameters needed to specify the lights as a coherent system, $I_{s}$. In the lower-left panel of Table 2 is the rewrite structure of an on-joint walker, needing only seven different vectors. The prominence for the walker interpretation of this system of lights is $\mathrm{P}=f\left(I_{i} / I_{s}\right)=f(1.86)$.

For the system of lights that represents an off-joint walker, the information load of the light configuration is higher, $I_{i}=15$. That is, specifying the movements of all of the lights independently requires the specifications of 15 complex vectors. When vector substitution is performed, the information load goes down only slightly, $I_{s}=11$. Thus, the prominence of this light array as a walker is considerably lower than that for an on-joint stimulus: $\mathbf{P}=f\left(I_{i} / I_{s}\right)=f(1.36)$.

\section{Two Qualifications}

Before proceeding, two things should be noted. First, these prominence values, when compared to Restle's (1979), are extremely low. This deflation is due to the fact that my adaptation of a coding theory has omitted such considerations as phase, wavelength,

\footnotetext{
${ }^{1}$ There are two possible interpretations of this notion. First, $\mathbf{M}(E) / 2$ could mean that the length of the pendulum is halved but that the angular excursion as measured at the pivot remains the same. Second, it could mean that the length is constant but that the angular excursion is halved. I am using the first interpretation in my notation.
} 
and the plane in which the movements take place. (Tilt and amplitude are specified in each vector.) Since wavelength and the picture plane are shared by all movements, and since all are nearly locked in perfect synchrony or $180^{\circ}$ out of phase, the prominence values for these displays would increase sharply with the addition of these factors. Roughly, this would multiply each $I_{i}$ by a factor of 4 but add to each $I_{s}$ only three (or slightly more) parameter specifications. Thus, prominence values for on-joint and off-joint walkers might easily burgeon to approximately $f(5.2)$ and $f(4.0)$, respectively. These values would be very high in Restle's system.

Second, and more important, Restle did not compare prominence values across stimuli; he compared them only within a given stimulus for different perceived configurations. This would be prudent for computational reasons alone, since $P$ values will automatically increase as the number of lights involved in the display increases. Beyond this, the comparison of prominence values across different arrays of dynamic light configurations could yield inaccurate, even meaningless, estimates of the relative perceived goodness of the displays. Despite these problems, I have chosen to compare prominence values across displays. The reasons are that the two types of displays have equal numbers of lights and that they were generated from the same base algorithm and are - in an abstract sense - the same stimuli.

Accepting the validity of my cross-stimulus comparisons, then, what is important to this discussion is not the magnitude of the prominence values but the difference between them. Regardless of what version of a coding theory is used, given that a walker is seen, the on-joint representation will be better than the off-joint representation. This can also be shown in terms of nestings within a tree structure. Such nestings are shown in the lower panels of Figure 2. Notice that the on-joint walker is a two-branch system and that the off-joint walker is a four-branch system. (In both cases the head is not considered a branch but is separated from the center of moment of the walker so as not to confuse them.) The two-branch system is clearly simpler than the four-branch one.

My point in this presentation is two-fold.
First, this adaptation of vector addition and coding theory is enlightening for the comparison of certain dynamic configurations and provides theoretical substance to my data and to a less radical version of JohnsonLaird and Wason's (1977) claim: Off-joint displays can be considered as poorer figures than on-joint displays and their prominence values are in line with experimental results. Second, this model, although in tenor directly adapted from Restle (1979), cannot possibly be correct as it stands. The reason for this will become clear in the discussion of a second demonstration.

\section{Demonstration 2: A Spatially Anomalous Walker}

Restle's (1979) model allows both the coding of movement and form. That is, although five parameters are necessary for the movements of particular lights, additional parameters may be added to code spatial relations among the moving lights (see Restle, 1979, pp. 6-7). Logically speaking, however, his coding of form and movement are orthogonal. That is, the coding of spatial separation seems not to effect the coding of movement and, to a large degree, vice versa. This would be of great benefit if aspects of figure and motion did not interact. However, it is the point of this demonstration to show that figural properties must be a part of movement description in a domain as complex as gait.

\section{Method}

Using the same base algorithm mentioned before, an 11-light dynamic configuration was generated. It was identical to those used by Cutting (1978a) for gender recognition in gait except in one aspect. Unlike those canonical walkers, this display is scrambled in the particular spatial locales of the points of light. Consider the right ankle as an arbitrary point of reference. By artificially displacing the other body parts but holding their absolute motion constant, bizarre arrangements were generated. For example, the head was placed below the ankle on the screen. Likewise, the right shoulder was placed below the ankle but considerably behind the locale of the head. The right elbow, on the other hand, was placed marginally above the ankle, and so forth. A static approximation to this anomalous walker is shown in the upper right-hand panel of Figure 3. As in Figure 2 , only the right half of the walker is displayed in the figure. Otherwise, the stimulus cycled and moved across the screen as previous stimuli did but without a ground line. 
This stimulus was shown on the Tektronix screen to six graduate students and staff members in the psychology department at Stanford University. All had seen my computer-generated gait displays before. All were asked simply to report what they saw.

\section{Results and Discussion}

None reported seeing the presence of a human walker. Responses split generally between descriptions of an abstract machine squirming cyclically across the screen, suggesting some Kandinsky twittering device, and descriptions of cyclically related but independent entities, suggesting something akin to a swarm of mechanical bees. Thus, no viewer reported that the display looked human, but all reported coherence. Data concerning the perceptual salience of this anomalous walker as compared to the canonical walkers was not obtained and would be difficult to interpret in any case. Here, again, one gets into difficulties making comparisons across displays, rather than within a display for different interpretations. It is my impression, however, and that of a few other viewers, that the canonical stimuli are far better as coherent and impressive stimulus events than is the anomalous display.

What I take to be important to this demonstration is that the movement parameters for this anomalous walker are exactly those for the canonical displays. The only thing changed is the relative physical placement of the lights, after the vectors have been computed. In other words, these vectors ordinarily presuppose the structure of a canonical walker, but they need not. Moreover, in a formal way, the coding model presented earlier does not. As it stands, the physical relations among vectors can be scrambled, as in this demonstration, without the coding of the array being changed. Since the perception (and by inference the perceptual salience) of the anomalous walker and the canonical walker are different, and since my codes for them are the same, I infer that my coding model must be revised to reflect the perceptual differences. Insofar as my model can be taken as a correct adaptation of the spirit of Restle's (1979) model, this demonstration seems to suggest a similar inadequacy in his current formulation: Interac-

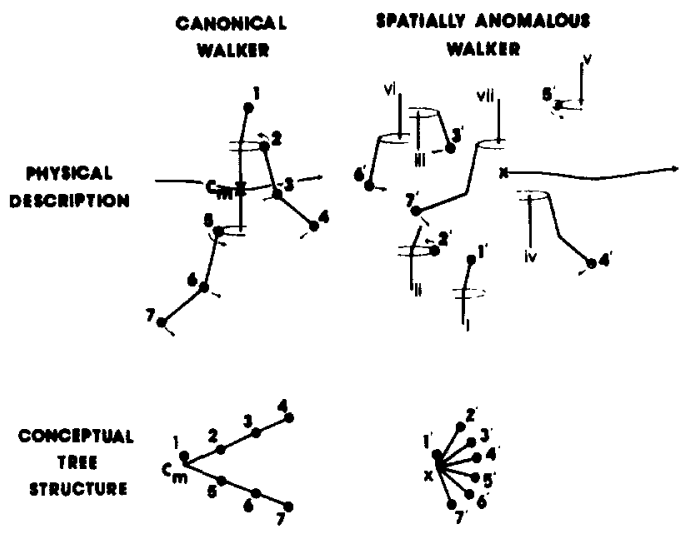

Figure 3. In the upper panels are the physical, vectorlike descriptions of a canonical walker and a spatially anomalous walker, one whose particular spatial relations among lights has been markedly perturbed; below these are conceptual tree structures of the nested movements around their generating centers. (Notice that only the canonical walker has a true center of moment; that for the anomalous walker is indeterminate in location.)

tions between spatial and dynamic relations may not be sufficiently dealt with. However, such a connection between this demonstration and Restle's model is tenuous. Thus, what follows is the necessary revision of my coding model, with a presently indeterminate relation to Restle's model. The crux of the change is the addition of a notion of nested centers of moment.

\section{A Second Model: Codes for Vectors and Their Generating Centers of Moment}

To capture both movement and spatial relations in a formal analysis of perceptual events, a new notation is needed. The one I propose is of the general form: $\mathrm{L}=\mathbf{M}(x$, $y)$, where $\mathrm{L}$ is movement of the particular light under consideration as a function of the movement $\mathbf{M}$ of point $x$ with respect to point $y$. The particular movement of any Light $\mathrm{L}$ can take the form of a circle, an ellipse, a pendulum arc, or more complex combinations, depending on the mechanical structure under consideration. The movement of point $x$ can be said to occur "around" point $y$. Thus, if $x$ describes a circle, $y$ is its center; if $x$ describes a pendulum arc, $y$ is the pivot; and if $x$ describes a corner of an oscillating flat spring in the shape of an isosceles trapezoid, $y$ is the oscillation center of that trap- 
ezoid. In each of these cases, $y$ can be considered the center for the movement of $x$; at all points within the trajectory of $x$, the point $y$ is its immediate referent. The general form of the equation, then, reads: The movement of Light $L$ can be described fully by the movement of point $x$ around point $y$.

Two things should be noted concerning the form of this model and its relation to other views. First, Restle's (1979) model implicitly has points moving about the center of a circle and thus would seem to account for centers of moment. What is crucial, however, is that he does not use this conception as a formal tool to relate certain nestings of the movements of different lights. Here I make this factor explicit. Second, Johansson (1958, $1973,1977)$, through his study of rigidity, motion, and spatial relations, has consistently expressed a view of event perception that can, for the most part, be fit snugly into this model. His approach, however, has been quite different, and not as formal.

The analysis that follows concerns two types of stimuli: One is an 11-light canonical stimulus used by Cutting (1978a), and the other is an 11-light, spatially anomalous walker as described in Demonstration 2. Schematic representations of these are shown in Figure 3. Lights on joints are renumbered as compared to Figure 2. Consider first the canonical walker.

\section{Coding of a Canonical Walker}

The movement of the light mounted on the head, 1, can be completely described as the movement of the highest order center of moment, $C_{m}$, with respect to some arbitrary point acting as ground, $\mathrm{g}$, plus whatever movement the head, $\mathrm{He}$, has with respect to the center of moment. In notational form, $1=\mathbf{M}\left(C_{m}, g\right)+\mathbf{M}\left(H e, C_{m}\right)$. Since the head does not move independently from the center of moment in my synthesis program, this equation reduces to: $1=\mathbf{M}\left(C_{m}, g\right)$.

Parametric description of the other lights proceeds in a similar fashion. For example, the light mounted on the wrist, 4 , is described by the four vector/center components of (a) the movement of the center of moment of the whole walker with respect to some arbitrary ground point plus (b) the movement of the shoulder with respect to the center of moment of the whole walker plus (c) the movement of the elbow with respect to the shoulder plus (d) the movement of the wrist with respect to the elbow. Since nearly all of these motions are phase locked in my synthesis routine, and in natural gait as well, phase of movement need not be considered here but could be incorporated into the model. This aspect of movement is important, and I will return to it in my concluding remarks. In addition, it should be noted that the center of moment for the whole walker lies between the shoulders and hips, but that all body parts move about their own local centers of moment. In other words, what I am calling the center of moment for the walker as a whole is the highest order, or most deeply nested, center in a system of centers. All body parts move in some way around this deepest point, but only the elbow and the wrist move about the shoulder, and only the knee and the ankle move about the hip. Thus, the shoulder and hip are the next deepest level of centers of moment. Likewise, the elbows and knees are the next level outward in this system of nested movements. What is shown in the upper left corner of Table 3 is a listing of the system of lights for a canonical walker. This is a grammatical system and as in Table 2 can be rewritten by vector/center substitutions. These are shown in the lower left panel of Table 3, and one graphic representation of these relations is shown in the lower left panel of Figure 3. This system is precisely that worked out elsewhere as a tree-structure hierarchy (Cutting \& Proffitt, in press, Figure 8).

In this particular version of a coding theory, the total possible information load in this canonical display is: $I_{l}=19$. Since it is perceived as a human walker, the load is considerably reduced, $I_{s}=7$. Thus, $\mathrm{P}=$ $f(19 / 7)=f(2.71)$. If considerations such as phase, wavelength, and plane are added, this value might easily jump to about $f(7.6)$.

\section{Coding of a Spatially Anomalous Walker}

In the upper-right panel of Figure 3, the seven points of light that would ordinarily represent the head and the joints of the right side of the body have been spatially discon- 
Table 3

Vector/Center Analyses for Computer-Generated Stimuli

\begin{tabular}{|c|c|}
\hline Canonical walker: $I_{i}=19$ & Spatially anomalous "walker": $I_{i}=19$ \\
\hline $1=\mathbf{M}\left(C_{m}, g\right)+\mathbf{M}\left(H e, C_{m}\right)=\mathbf{M}\left(C_{m}, g\right)$ & $1^{\prime}=\mathbf{M}(x, g)+\mathbf{M}(H e, i)=\mathbf{M}(x, g)$ \\
\hline $2=\mathbf{M}\left(C_{m}, g\right)+\mathbf{M}\left(S, C_{m}\right)$ & $2^{\prime}=\mathbf{M}(x, g)+\mathbf{M}(S, i i)$ \\
\hline $3=\mathbf{M}\left(C_{m}, g\right)+\mathbf{M}\left(S, C_{m}\right)+\mathbf{M}(E, S)$ & $3^{\prime}=\mathbf{M}(x, g)+\mathbf{M}\left(S^{\prime}, i i i\right)+\mathbf{M}\left(E, S^{\prime}\right)$ \\
\hline $4=\mathbf{M}\left(C_{m}, g\right)+\mathbf{M}\left(S, C_{m}\right)+\mathbf{M}(E, S)+\mathbf{M}(W, E)$ & $4^{\prime}=\mathbf{M}(x, g)+\mathbf{M}\left(S^{\prime \prime}, i v\right)+\mathbf{M}\left(E^{\prime}, S^{\prime \prime}\right)+\mathbf{M}\left(W, E^{\prime}\right)$ \\
\hline $\mathbf{S}=\mathbf{M}\left(C_{m}, g\right)+\mathbf{M}\left(H i, C_{m}\right)$ & $5^{\prime}=\mathbf{M}(x, g)+\mathbf{M}(H i, v)$ \\
\hline $6=\mathbf{M}\left(C_{m}, g\right)+\mathbf{M}\left(H i, C_{m}\right)+\mathbf{M}(K, H i)$ & $\mathbf{6}^{\prime}=\mathbf{M}(x, g)+\mathbf{M}\left(H i^{\prime}, v i\right)+\mathbf{M}\left(K, H i^{\prime}\right)$ \\
\hline $7=\mathbf{M}\left(C_{m}, g\right)+\mathbf{M}\left(H i, C_{m}\right)+\mathbf{M}(K, H i)+\mathbf{M}(A, K)$ & $7^{\prime}=\mathbf{M}(x, g)+\mathbf{M}\left(H i^{\prime \prime}, v i i\right)+\mathbf{M}\left(K^{\prime}, H i^{\prime \prime}\right)+\mathbf{M}\left(A, K^{\prime}\right)$ \\
\hline $\begin{array}{l}\text { Reduced code by vector/center } \\
\text { substitution: } I_{s}=7\end{array}$ & $\begin{array}{l}\text { Reduced code by vector/center } \\
\text { substitution: } I_{s}=13\end{array}$ \\
\hline $\mathrm{P}=f\left(I_{i} / I_{s}\right)$ & $\mathrm{P}=f\left(I_{i} / I_{s}\right)$ \\
\hline$=f(2.71)$ & $=f(1.46)$ \\
\hline $1=\mathbf{M}\left(C_{m}, \mathbf{g}\right)$ & $1^{\prime}=\mathbf{M}(x, g)$ \\
\hline $2=1+\mathbf{M}\left(S, C_{m}\right)$ & $2^{\prime}=1^{\prime}+\mathbf{M}(S, i i)$ \\
\hline $3=2+\mathbf{M}(E, S)$ & $3^{\prime}=1^{\prime}+\mathbf{M}\left(S^{\prime}, i i i\right)+\mathbf{M}\left(E, S^{\prime}\right)$ \\
\hline $4=3+M(W, E)$ & $4^{\prime}=1^{\prime}+\mathbf{M}\left(S^{\prime \prime}, i v\right)+\mathbf{M}\left(E^{\prime}, S^{\prime \prime}\right)+\mathbf{M}\left(W, E^{\prime}\right)$ \\
\hline $5=1+\mathbf{M}\left(H i, C_{m}\right)$ & $5^{\prime}=1^{\prime}+\mathbf{M}(H i, v)$ \\
\hline $6=5+\mathbf{M}(K, H i)$ & $6^{\prime}=1^{\prime}+\mathbf{M}\left(H i^{\prime}, v i\right)+\mathbf{M}\left(K, H i^{\prime}\right)$ \\
\hline $7=6+\mathbf{M}(A, K)$ & $7^{\prime}=1^{\prime}+\mathbf{M}\left(H i^{\prime \prime}, v i i\right)+\mathbf{M}\left(K^{\prime}, H i^{\prime \prime}\right)+\mathbf{M}\left(A, K^{\prime}\right)$ \\
\hline
\end{tabular}

Note. $\mathbf{M}=$ movement of point following it; $C_{m}=$ highest order center of moment; $H e=$ head; $S=$ shoulder; $E$ = elbow; $W=$ wrist; $H i=$ hip; $K=$ knee; $A=$ ankle, $g$ is an arbitrary ground point. Arabic numbers refer to movements of lights shown in Figure 3; lowercase italicized Roman numerals refer to arbitrary, unrelated points in space, as shown in the upper right panel of Figure 3. Primed values of joints indicate that the locations are different for each occurrence of a particular joint. In the general form of the notation, $\mathbf{M}(x, y), I$ am considering the movement of point $x$ with respect to point $y$, a mechanically determinable point based on the vector structure of $x . I_{i}=$ total number of vector/center doubles needed to specify the lights independently; $I_{s}=$ total number of vector/center doubles needed to specify the lights as a coherent system. $P$ is the prominence of the perceived configuration, as a function $(f)$ of $I_{i}$ divided by $I_{s}$.

nected. In the demonstration, these (and the lights mounted on the left side of the body) all move across the screen. They share the common vector of motion that corresponds in the canonical walker to $\mathbf{M}\left(C_{m}, g\right)$. This time, however, since the array is spatially disorganized, there is no determinable highest order center of moment. Thus, lights share an undulatory translation vector of some unknown point $x$, and the vector movement can only be described as $\mathbf{M}(x, g)$, the movement of this unknown point with respect to some arbitrary ground point $g$. This vector is shared by all seven points, but nothing else is shared. In particular, although the seven points labeled $i$ and $v i i$ are the same point in the canonical walker, they bear no systematic relation to one another here.
Thus, they cannot form the basis of a nesting scheme. In the vector/center substitution carried out in the lower-right section of Table 3, the movement of Light 2', for example, cannot be substituted into the movement of Light $3^{\prime}$ because the local centers of moment for the two are not shared, one being point $i i$ and the other being point iii. The same nonsubstitutability holds for Lights $3^{\prime}$ and $4^{\prime}, 5^{\prime}$ and $6^{\prime}$, and $6^{\prime}$ and $7^{\prime}$. Thus, the grammar for this spatially anomalous walker is entirely different, conceptually shown in the lower right panel of Figure 3 . This is to be compared against that for the canonical walker in the lower-left panel of the same figure.

This adaptation of coding theory suggests that the total possible information load of 
this display is the same as the canonical walker: $I_{i}=19$. However, vector-center substitutions allow the reduction of this parameter load, $I_{s}=13$. Thus, $\mathrm{P}=f(19 / 13)=13$. Thus, $\mathrm{P}=f(19 / 13)=f(1.46)$. If considerations of phase, wavelength, and plane are added, this value jumps to about $f(4.15)$.

Given these values for the two walkers, two facts become important. First, computed prominence for the spatially anomalous walker is considerably below that for the canonical walker. This suggests that the canonical figure is a much better one-as indeed the demonstration indirectly suggested. Second, the previous version of my coding model does not discriminate between the two types of walkers. Both would have prominence values of $f(2.71)$ by my conservative method of calculation and near $f(7.6)$ by considering the other shared parameters.

In addition, although codes get slightly more complex, prominence values for onand off-joint stimuli used in Experiment 1 remain the same. Thus, this second model includes the first as a special case. Moreover, the second model should be applicable to events other than walking and should be useful as a descriptive and predictive device.

\section{Discussion}

This adaptation of a coding theory to event perception was motivated by an attempt to make mutually comparable Restle's (1979) formal model and my colleagues and my less formal account of centers of moment. I have preserved Johansson's (1973) vector-analytic approach in part because Restle's five movement parameters do not distinguish circular motion from pendular motion, or any other type of movement. Thus, instead of parameters I have used complex vectors as primitives within the coding system. This has a certain degree of inelegance, but when dealing with biological movements, a microanalysis of complex vectors seems not only overwhelmingly complicated, but also only tangentially relevant.

\section{Perception and Coding}

The major thrust of this article is to suggest that both movements and spatial rela- tions are necessary to any formal account of event perception and that these must interact. In particular, the coding of nested motions of various parts of a dynamic figure must include the coding of points from which the motions are generated. More concretely, a coding model, regardless of its form, should be able to account for the two types of results suggested in this article: It must provide a rationale for why on-joint representations of human walkers are better than off-joints representations and why a canonical display of a walker is much better than a spatially anomalous one.

My second model does this in the following way: Consider first the on-joint and offjoint stimuli. Both of these displays have a highest order center of moment. The location of this point lies roughly midway between the shoulder and the hip. Only the on-joint display, however, has a nested hierarchy of such centers, with the shoulder and hip serving as local centers of moment for the wrist and ankle, respectively. This nesting provides a simple and straightforward means of vectorial decomposition to find the highest order center of moment of the display. The method outlined later proceeds in an order suggested by Wallach (1965) and is opposite to that suggested by Johansson (1973). (See Cutting \& Proffitt, in press, Proffitt \& Cutting, 1979, and Proffitt et al., 1979, for the rationale of the ordering.)

One possible plan for perceiving an onjoint stimulus is as follows: First, the movements of the wrist and shoulder are compared, and by subtracting out the relative vector, one obtains redundant and more salient information about the movement of the shoulder. Likewise, comparing the movements of the ankle and hip and subtracting out the relative vector, one obtains redundant and more salient information about the movement of the hip. Finally, comparing the residual common vectors for all four lights mounted on the right side of the body, together with those of the wrist and ankle on the left side and with that of the head, the locus and movement of the highest order center of moment fall out as residuals. In our analysis, the locus and movement of this point are strongly correlated information that can be used to make accurate gender 
judgments (Cutting, 1978a; Cutting et al., 1978). Thus, it makes a certain amount of sense that the on-joint figures, and by extension the canonical ones, should make good figures with high prominence values, allowing gender identification.

The off-joint display, on the other hand, provides a not-so-simple and not-so-straightforward vector decomposition. Comparing the movements of a light mounted on the right biceps and right forearm does not provide redundant information about a visible point in the display. Instead, that point is an invisible one, the shoulder. Likewise, comparing the movements of lights mounted on the thigh and calf have redundancy for the unseen hip. Amalgamating the residual movements from all of the points around the body leaves the perceptual system with the task of deriving to locus and movement of an unseen point (the highest order center of moment) from the locus and movements of two other unseen points (the shoulder and hip, which are more local centers of moment). Conceptually, this seems like a more difficult task and to the extent that this logic reflects what the perceptual system is doing, it makes sense that gender judgments should be more difficult in this case. In fact, with no experience, viewers in Group 3 of Experiment 1 could not perform the task at all, but with a few minutes experience, those in the other groups could judge gender moderately well, though never as well as from on-joint or canonical displays.

The accounts for the difference in perception between the canonical and anomalous displays proceed in a similar fashion. The logic for the canonical display is simply an extension and elaboration of that for the onjoint stimulus given earlier, so it need not be repeated here. The logic for anomalous display is as follows: It makes little sense that limbs of the human body should be isolated, since viewers did not report seeing a humanlike structure in the display. Thus, perception would probably follow something like an adjacency principle (Gogel, 1978). For any pairwise comparison of lights, the relative vector would subtract out, since all residual motion would be the same. However, all residuals would leave the perceptual system with no consistent center of moment for the display as a whole. The fact that there is no center of moment may have contributed to the perception of this conflation of lights by some viewers as not a single entity but a swarm of closely related entities. The display obviously had coherence, since all points shared a phase-locked undulatory vector across the screen, but there was no central structure in that coherence. Thus, the lights had a common fate but no nested common fate about a common center. The nesting shown in the lower right-hand panel of Figure 3 is, I would argue, precisely the nesting that is necessary for the specification of a human figure.

\section{Dynamics, Kinetics, and Kinematics}

The study of mechanics divides several ways, and this parsing seems likely to be relevant to the perception of events. First, statics is contrasted with dynamics. Statics, on the one hand, deals with unmoving bodies and the equilibrium of forces, and dynamics, on the other hand, deals with moving bodies and with the action of forces that move them. Dynamics is subdivided because the treatment of moving bodies in themselves is called kinematics, whereas the treatment of moving bodies as a result of forces played on them is called kinetics.

These distinctions are important because the coding theory approaches used here and in Restle (1979) are both kinematic. That is, neither refers in any overt manner to the forces that generate motion-both are studies in pure movement. I would expect, however, that the approach used here will prove consistent with a kinetic approach. The reason for this assertion is that it seems to me that the concept of centers of moment is neutral with respect to the distinction within dynamics. Thus, centers of moment should prove equally applicable to kinematics and kinetics. I would expect further that kinetic approaches to event perception will prove superior to kinematic ones (see Runeson \& Frykholm, in press). After all, forces are determined by mass times acceleration. When perceiving gait, for example, we perceive the movements of torso, arms, and legs, each of which have accustomed masses and which accelerate according to known laws. 
These movements are locked in time to these masses and forces. To change artifically the relative speed of a walker, as done by Barclay, Cutting, and Kozlowski (1978, Experiment 2) is to disrupt the whole kinetic process, leaving the kinematic description intact. Since such changes in presentation rate disrupt gait perception, it is likely that a kinetic approach to gait, and by extension to other events, will prove more efficacious.

\section{Some Caveats}

There are several other crucial components of motion not formally considered in my system. In particular, phase and the plane in which the movement takes place are omitted here but included by Restle (1979). These are serious omissions in my formulation. Consider each in turn.

Phase is extremely important in all biological motion. Phase is precisely what separates walking from running in bipedal locomotion and walking, pacing, trotting, and galloping in quadrupedal gait. Moreover, slight asynchronies in phase are already present in my synthetic walker program (Cutting, 1978c), which I have not discussed. In particular, the movement of the ankle with respect to the knee is not quite in phase, and to place them artificially in phase is to create an unnatural looking gait, one of toe stubs occurring with each step.

The plane in which movement takes place is also crucial. Although assuming a single plane for walker movement is a useful simplification, it ignores the potential richness possible in human biomechanics. In particular, my notation would be inadequate for dancing or for sign language precisely because in those domains many planes are used. In fact, my system, like Restle's (1979), ignores the fundamental three-dimensionality of space and some of the richness offered by Johansson's (1977) analyses of projective geometry.

Phase and planar considerations could be added to this notation system but at the expense of clarity and simplicity. Thus, I have not attempted to add them here. Instead, the force of my presentation is that mechanical movements in a nested system are not only vectorally coherent but also spatially coher- ent around movement centers. That is, movements are generated from specific places, like the joints of a walker, and these nested centers of moment are crucial to this type of event perception.

\section{Reference Note}

1. Johansson, G. Visual perception of biological motion and a model analysis (Report 100). Uppsala, Sweden: University of Uppsala, Department of Psychology, 1971.

\section{References}

Barclay, C. D., Cutting, J. E., \& Kozlowski, L. T. Temporal and spatial factors in gait perception that influence gender recognition. Perception \& Psychophysics, 1978, 23, 145-152.

Cutting, J. E. Generation of synthetic male and female walkers through manipulation of a biomechanical invariant. Perception, 1978, 7, 393-405. (a)

Cutting J. E. Perceiving the geometry of age in a human face. Perception \& Psychophysics, 1978, 24, 566568. (b)

Cutting, J. E. A program to generate synthetic walkers as dynamic point-light displays. Behavior Research Methods \& Instrumentation, 1978, 10, 91-94. (c)

Cutting, J. E., \& Kozlowski, L. T. Recognizing friends by their walk: Gait perception without familiarity cues. Bulletin of the Psychonomic Society, 1977, 9, 353-356.

Cutting, J. E., \& Proffitt, D. R. Gait perception as an example of how we may perceive events. In H. L. Pick \& R. Walk (Eds.), Perception and perceptual development (Vol. 2). New York: Plenum Press, in press.

Cutting, J. E., Proffitt, D. R., \& Kozlowski, L. T. A biomechanical invariant for gait perception. Journal of Experimental Psychology: Human Perception and Performance, 1978, 4, 356-372.

Gogel, W. C. The adjacency principle in visual perception. Scientific American, 1978, 238(5), 126-138.

Johansson, G. Configurations in event perception. Uppsala, Sweden: Almquist \& Wiksell, 1950.

Johansson, G. Rigidity, stability, and motion in perceptual space. Acta Psychologica, 1958, 14, 359-370.

Johansson, G. Visual perception of biological motion and a model for its analysis. Perception \& Psychophysics, 1973, 14, 201-211.

Johansson, G. Visual motion perception. Scientific American, 1975, 232(6), 76-89.

Johansson, G. Spatial constancy and motion is visual perception. In W. Epstein (Ed.), Stability and constancy in visual perception. New York: Wiley, 1977.

Johansson, G., von Hofsten, C., \& Jansson, G. Event perception. Annual Review of Psychology, 1980, 31, 27-63.

Johnson-Laird, P. H., \& Wason, P. C. (Eds.), Thinking: Readings in cognitive science. Cambridge, England: Cambridge University Press, 1977.

Kozlowski, L. T., \& Cutting, J. E. Recognizing the sex of a walker from a dynamic point-light display. Perception \& Psychophysics, 1977, 21, 575-580. 
Leeuwenberg, E. L. J. A perceptual coding language for visual and auditory patterns. American Journal of Psychology, 1971, 84, 307-349.

Leeuwenberg, E. L. J. Quantification of certain visual pattern similarities: Salience, transparency, similarity. In E. L. J. Leeuwenberg \& H. F. J. M. Buffart (Eds.), Formal theories of visual perception. New York: Wiley, 1978.

Marey, E. J. Movement. New York: Arno Press \& New York Times, 1972. (Originally published, 1895.)

Murray, M. P. Gait as a total pattern of movement. American Journal of Physical Medicine, 1967, 46, 290-333.

Proffitt, D. R., \& Cutting, J. E. Perceiving the centroid of configurations on a rolling wheel. Perception \& Psychophysics, 1979, 25, 389-398.
Proffitt, D. R., Cutting, J. E., \& Stier, D. M. Perception of wheel-generated motions. Journal of Experimental Psychology: Human Perception and Performance, 1979, 5, 289-302.

Restle, F. Coding theory of the perception of motion configurations. Psychological Review, 1979, 86, 1-24.

Runeson, S. \& Frykholm, G. Visual perception of lifted weight. Journal of Experimental Psychology: $\mathrm{Hu}$ man Perception and Performance, in press.

Sober, E. Simplicity. New York: Oxford University Press, 1975.

Wallach, H. Visual perception of motion. In G. Kepes (Ed.), The nature and art of motion. New York: George Braziller, 1965.

Received August 2, 1979

\section{Instructions to Authors}

For further information on content, authors should refer to the editorial in the August 1978 issue of the Journal (Vol. 5, No. 3, pp. 355-356). Authors should prepare manuscripts according to the Publication Manual of the American Psychological Association (2nd ed.). Instructions on tables, figures, references, metrics, and typing (all copy must be doublespaced) appear in the Manual. Manuscripts should include an abstract of 100-175 words. Authors are requested to refer to the "Guidelines for Nonsexist Language in APA Journals" (Publication Manual Change Sheet 2, American Psychologist, June 1977, pp. 487-494) before submitting manuscripts to this journal.

Authors should submit manuscripts in quadruplicate, and all copies should be clear, readable, and on paper of good quality. Authors should keep a copy of their manuscript to guard against loss. Mail manuscripts to the Acting Editor, Margaret Jean Intons-Peterson, Department of Psychology, Indiana University, Bloomington, Indiana 47405.

Addresses for the editors of the other JEP journals are as follows: Journal of Experimental Psychology: General, Gregory A. Kimble, Department of Psychology, Duke University, Durham, North Carolina 27706 (submit 4 copies of the manuscript); Journal of Experimental Psychology: Human Learning and Memory, Richard M. Shiffrin, Department of Psychology, Indiana University, Bloomington, Indiana 47405 (submit 4 copies of the manuscript); and Journal of Experimental Psychology: Animal Behavior Processes, Allan R. Wagner, Department of Psychology, Yale University, New Haven, Connecticut 06520 (submit 3 copies of the manuscript).

The editors have agreed to use blind review when it is requested by the author. Authors requesting blind review should prepare manuscripts to conceal their identity. 